\title{
Obstetric Outcome in Women with Intrahepatic Cholestasis: A 3-year Study in a Tertiary Care Hospital in Bengaluru
}

\author{
Vishnupriya KM Naga ${ }^{1}$, Biji Joseph ${ }^{2}$, Manjula S Kalappa ${ }^{3}$
}

\begin{abstract}
Objective: To study the incidence and obstetric outcome of pregnancies complicated by intrahepatic cholestasis.

Materials and methods: A retrospective case control study of 60 women with obstetric cholestasis was carried out in the Department of Obstetrics and Gynecology, St. John's Medical College Hospital, Bengaluru from January 2015 to March 2018. Statistical analysis was performed using the $\mathrm{Z} / \mathrm{T}$ test when appropriate. A $p$ value less than or equal to 0.05 was considered statistically significant.

Results: The frequency of intrahepatic cholestasis of pregnancy (ICP) was 60 among 8319 deliveries (7.21\%). The most common symptom was generalized pruritus, which appeared in $36.6 \%$ during the 2 nd trimester and $83.3 \%$ in the 3 rd trimester of cases. Preeclampsia was seen in $31.6 \%$ (3.3\% in controls); preterm deliveries were seen in ( $23 \%$ vs $5 \%$ ). A higher incidence of meconium-stained amniotic fluid ( $25 \%$ vs $6.7 \%$ ) was noted at delivery. The cesarean section rate was $41.7 \%$ vs $23.3 \%$. There was no significant difference in the CTG changes, APGAR score less than 7 , intrauterine growth restriction, NICU admission or perinatal mortality between cases and controls.

Conclusion: Multidisciplinary approach involving obstetricians, gastroenterologists and neonatologists improved the obstetric outcome. Early diagnosis was done with liver function tests (aminotransferases) and urinary bile salts and bile pigments. In most of the cases, antihistaminics and emollients relieved the symptoms. Ursodeoxycholic acid (UDCA) treatment is found to be effective in reducing the persistent pruritus.

Keywords: Intrahepatic cholestasis, Maternal outcome, Perinatal outcome, Ursodeoxycholic acid.

Journal of South Asian Federation of Obstetrics and Gynaecology (2019): 10.5005/jp-journals-10006-1662
\end{abstract}

\section{INTRODUCTION}

Intrahepatic cholestasis of pregnancy (ICP)—also known as obstetric cholestasis $(\mathrm{OC})$ - is a unique liver disease of pregnancy, which is associated with pruritus involving palms and soles without any skin rash ${ }^{1}$ and with increased liver enzymes. ${ }^{2}$ It is typically seen in the late second and third trimester ${ }^{3,4}$ although some studies have reported presentation as early as 6- to 10 -weeks gestation. ${ }^{5-7}$ The itching subsides almost immediately after delivery ${ }^{8-10}$ and the serum bile acid levels and the liver enzymes normalize within 2-3 weeks of delivery. ${ }^{8}$

The incidence varies from 0.1 to $15.6 \%$ all over the world. It is found to be $0.1-1.5 \%$ in Central/Western Europe and North America; and $1.5-4 \%$ in Chile and Bolivia. ${ }^{11}$ ICP is more common in the winter months in Finland, Sweden, Chile, and Portugal. A higher incidence is seen in twin pregnancies. ${ }^{12}$

Cholestasis is classified as intrahepatic or extrahepatic. Intrahepatic cholestasis primarily involves the bile canaliculi and the intrahepatic bile ducts. Extrahepatic cholestasis involves the extra hepatic ducts, the common hepatic duct, or the common bile duct.

The pathogenesis of ICP is multifactorial and includes genetic, hormonal, and environmental factors. ${ }^{13}$ Studies have demonstrated mutations in the $A B C B 4$ gene, leading to abnormalities in the MDR3 protein in approximately $16 \%$ of patients with ICP. Such mutations lead to dysfunction of bile transport across the canaliculus. Hormonal factors also play a role. There is a greater incidence of ICP in twin pregnancies (where peak estrogen levels are higher) than in singleton pregnancies. ICP is most commonly observed in the third trimester as the serum concentration of estrogen is at the highest level. The significance of cholestasis of pregnancy is associated with a high incidence of perinatal complications, such as meconium-stained liquor, fetal distress, nonreactive NST,
${ }_{1-3}$ Department of Obstetrics and Gynecology, St. John's Medical
College Hospital, Bengaluru, Karnataka, India

Corresponding Author: Manjula S Kalappa, Department of Obstetrics and Gynecology, St. John's Medical College Hospital, Bengaluru, Karnataka, India, Phone: +91 9742266558, e-mail: thatva@rediffmail.com How to cite this article: Naga VKM, Joseph B, et al. Obstetric Outcome in Women with Intrahepatic Cholestasis: A 3-year Study in a Tertiary Care Hospital in Bengaluru. J South Asian Feder Obst Gynae 2019;11(2): 103-106.

Source of support: Nil

Conflict of interest: None

decreased fetal movement, and prematurity. The treatment with ursodeoxycholic acid (UDCA) could decrease maternal bile acid concentrations and improve fetal outcomes. ${ }^{14,15}$ It also reduces the itching and normalizes the liver functions in ICP. ${ }^{16}$ UDCA can also decrease toxic endogenous bile acids by placental transfer of bile acids, thus lowering the amount of bile acids present in the colostrum. ${ }^{17,18}$

\section{Materials and Methods}

A retrospective case control study was conducted at the Department of Obsterics and Gynaecology, St. John's Medical College Hospital, Bengaluru. After obtaining the ethicalcommittee's clearance, the women with ICP who delivered between 2015 January and 2018 March were taken as cases and the immediate next patient details were taken as controls. The diagnosis of ICP was made by reviewing all the case records for patient profile, complaints, associated medical and obstetric complications, investigations, and treatment. Pregnancy-specific ranges of LFT s were used. (AST/ALT-more than 
35 and 25, respectively). For the transaminases, gamma glutamyl tranferase (GGT), and bilirubin in pregnancy, the upper limit of normal value is $20 \%$ lower than that of nonpregnant state. Alkaline phosphate (ALP) is found to rise normally in pregnancy and is considered abnormally high if there is at least a threefold increase over the nonpregnant normal value.

The exclusion criteria were chronic liver diseases like hepatitis B, hepatitis $C$, primary biliary cirrhosis, primary sclerosing cholangitis, symptomatic cholelithiasis, Wilson's disease, acute fatty liver of pregnancy (AFLP) and HELLP syndrome (haemolysis, elevated liver enzymes, and low platelets).

The obstetric complications, mode of delivery, and perinatal outcome of cases were compared with controls. Women diagnosed with ICP were followed up regularly with liver function tests (LFT) every 2 weeks. Fetal surveillance was done with daily fetal movement count (DFMC), and twice weekly Non stress test (NST) at 34 weeks of GA, and sonography for amniotic fluid index (AFI) every 7-14 days based upon gestation. The control group had regular antenatal visits, as well as NST and sonography whenever indicated.

The complications were decreased fetal movements, preeclampsia, preterm delivery, meconium stained liquor, and the need for NICU admissions.

\section{Results}

During the study period, there were 8319 deliveries, of which 60 (7.21\%) were diagnosed with ICP. The mean age of these women was $27.37 \pm 3.8$ years in cases and $25.2 \pm 4.5$ years in controls. Among these $68.3 \%$ were primi and $31.7 \%$ were multi (In controls $58.3 \%$ and $41.7 \%$ ). (Fig. 1).

Generalized pruritus (including palms and soles [100\%])—which worsened at night, causing sleep deprivation-was the principal symptom seen in all these women. In most of these women, symptoms started at the end of the second trimester in $37 \%$ or at the starting of the third trimester in $63 \%$ of cases (Fig. 2).

Using pregnancy-specific changes for the LFT, it was found that the amount of transaminases was the most elevated (85\%) in the study group. Urine bile salts and pigments were found to be positive in $8.3 \%$ of cases (Fig. 3).

All were treated symptomatically with antihistaminics and emollients and only 30\% required UDCA in a dose of 300-600 mg/day, in patients with persistent pruritis. The obstetric

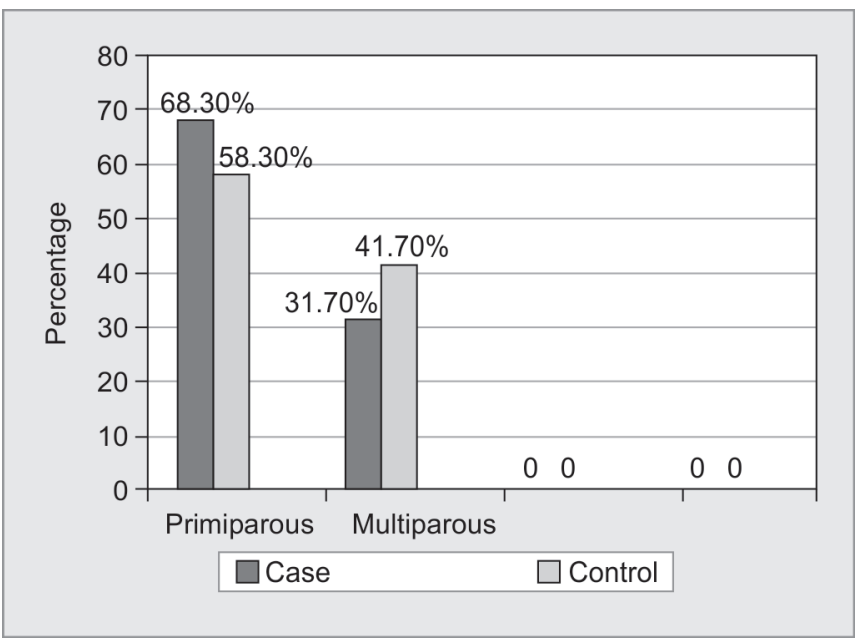

Fig. 1: General profile of patients complications noted were mild preeclampsia $31.6 \%$ vs $3.3 \%$ and GDM $20 \%$ vs $16 \%$; gestational hypertension was found in $6.6 \%$ vs $5 \%$ of the cases. Hyperbilirubinemia was present in $5 \%$ of cases. And $23 \%$ vs $5 \%$ had preterm delivery and $1.6 \%$ had PPH (Fig. 4).

Of the 60 cases, $28.3 \%$ delivered spontaneouly at term. An estimated $71.7 \%$ of the cases were induced, out of which $30 \%$ delivered by FTVD and $41.7 \%$ by cesarian sections (in controls $76.7 \%$ and $23.3 \%$ ). The indications for LSCS were as follows: $71.6 \%$ had fetal distress (controls 56\%), 16\% had CPD, and 16\% had failed induction, and $12 \%$ had NPL (nonprogression of labour). All these study groups were delivered between 37 and 38 weeks of gestational age and the control group was delivered between 39 and 40 weeks (Fig. 5).

The perinatal complications were meconium-stained liquorwas seen in $25 \%$ vs $16.7 \%$, Fetal distress/abnormal CTG—was seen in $71.6 \%$ vs $56 \%$, IUGR - was seen in $52.6 \%$ vs $47.3 \%$, and $31.7 \%$ vs $18.3 \%$ babies had to be admitted to NICU (Fig. 6).

\section{Discussion}

Intrahepatic cholestasis of pregnancy is a reversible form of cholestasis that is characterized by an impaired bile flow that occurs in the late second and third trimester of pregnancy. It resolves rapidly after delivery. ${ }^{19}$ In our study, the incidence was of $7.2 \%$, and it is attributed to the tertiary referral hospital. Incidence in India has been reported to be $1 \%$.

The mean age of the patients in our study group was 27.5 years, and $68.3 \%(n=41)$ were primipara and $31.7 \%(n=19)$ were multiparous, which is comparable to those reported by Padmaja et al. ${ }^{20}$ Generalized pruritis was the cardinal symptom in all patients, which is more pronounced in the palms and soles, which was similar to that observed by Kenyon et al.

In our study there were no statistically significant clinical or biochemical predictors associated with an increased risk of fetal complications. This may in part be due to the difference in clinical practice: we delivered patients with diagnosed ICP at 37-38 weeks of gestational age.

ICP is associated with abnormalities of the transaminases, GGT, bilirubin, and urine bile salts. ${ }^{20}$ In our study, $85-86 \%(n=52)$ of the cases had elevated transaminases.

In our study, $5 \%(n=3)$ had hyper bilirubinemia and there was no case of jaundice, when compared to another study, which showed $18.4 \%$ of hyperbilirubinemia. ${ }^{20}$

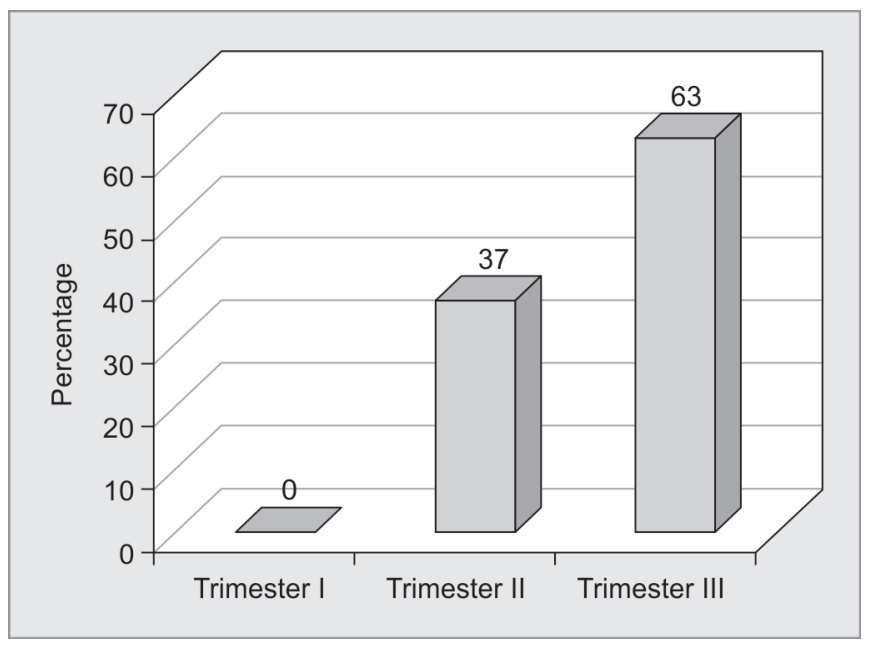

Fig. 2: Gestational age at diagnosis in obstetric cholestasis 


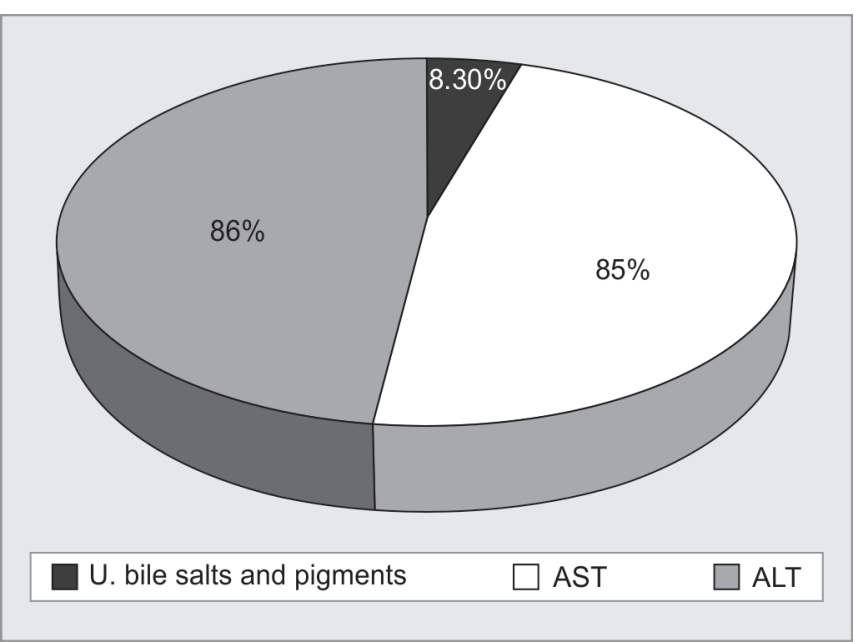

Fig. 3: Diagnostic parameters

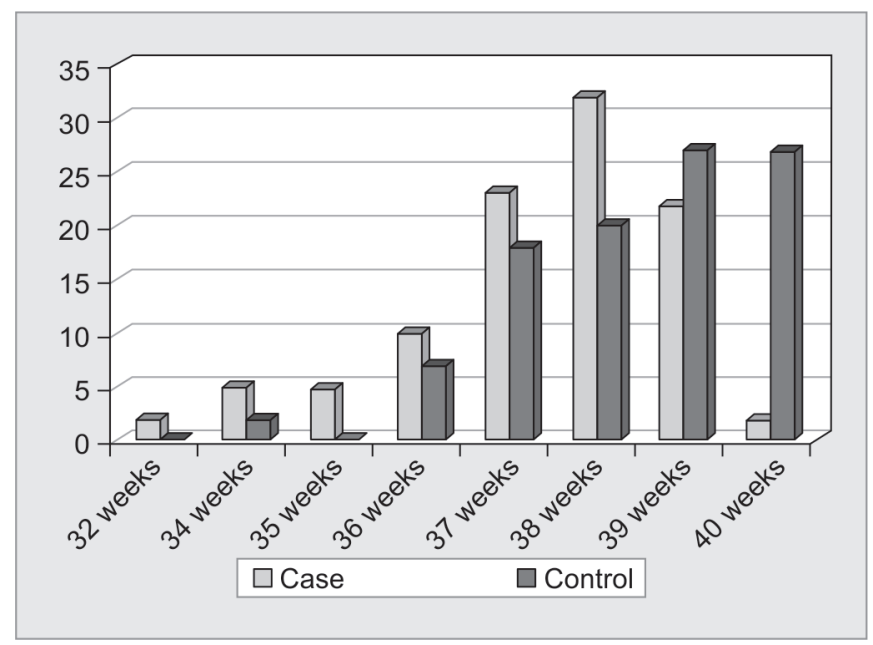

Fig. 5: Gestational age at delivery in obstetric cholestasis

Even though ICP is benign to the mother, it is associated with increased fetal complications. In our study we found that $44.5 \%$ of birth had been associated with perinatal complications, including meconium-stained amniotic fluid, fetal distress, nicu admissions, and there were no episodes of intrauterine fetal death, comparing to the Rook et al. study, which showed $33 \%$ perinatal complications. The incidence of preterm delivery in our study was 23\% ( $n=14)$ compared to $5 \%(n=3)$ in controls, whereas Padmaja et al. study showed the rate of preterm delivery is $24 \%$ of cases. ${ }^{20}$

The mechanisms of preterm delivery in ICP is not clear, but studies show an increased response of myometrial strips from healthy women to oxytocin and an increased oxytocin receptor expression after being incubated with cholic acid. ${ }^{21}$

In our study there were no IUD, but Glanz et al. ${ }^{7}$ have showed intrauterine demise in $0.4 \%$ of ICP. The incidence of meconium staning is $25 \%(n=15)$ in our study, which is in accordance with that observed in Padmaja et al. study (24\%). ${ }^{20}$ In our study the incidence of PPH was found to be high in women with ICP $1.6 \%(n=1)$ who did not receive $\mathrm{Vit} K$, compared to who received vit $\mathrm{K}$ according to Keneyon et al.

Our study had several limitations. Since it was a retrospective study, it was difficult to control the missing data. The majority of the patients did have AST/ALT and urine bile salts and bile pigments'

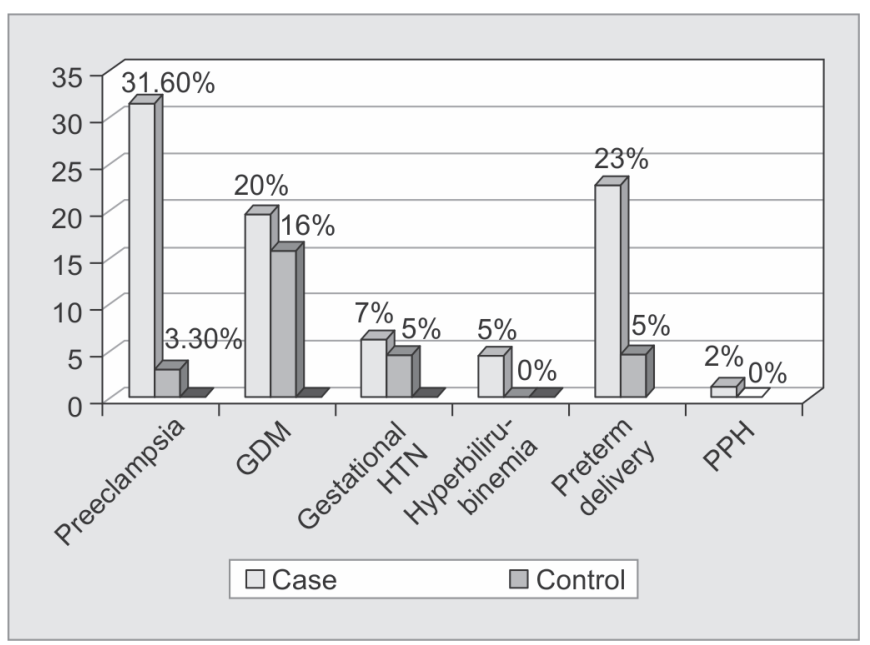

Fig. 4: Obstetric complications

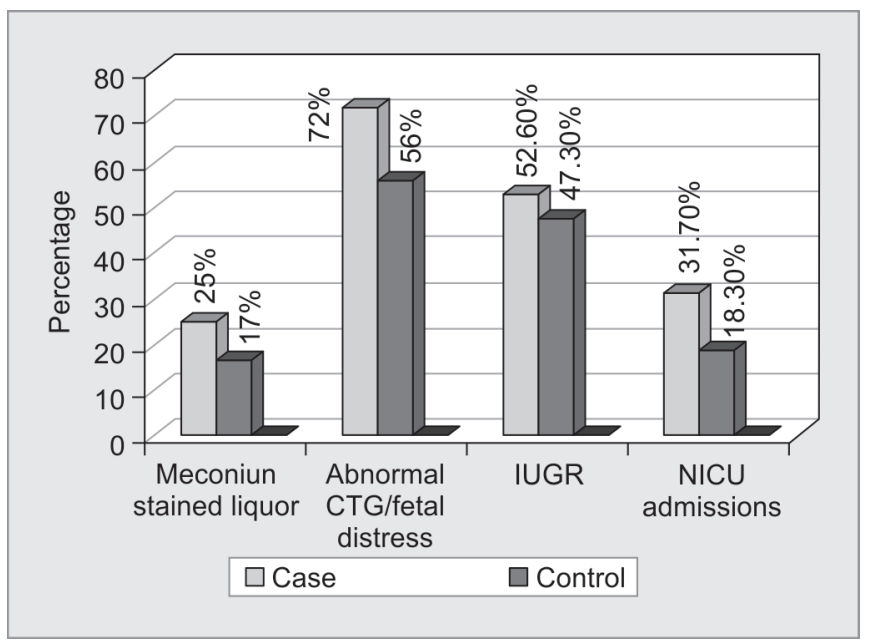

Fig. 6: Perinatal outcome

report. However, not all women were tested for other causes of potential liver disease. The criteria for diagnosing ICP are also subjective, as there are no current uniform criteria for the diagnosis of ICP. Most studies used elevated serum bile acids' or serum transaminases' level combined with pruritus during pregnancy. However, the serum bile acids may not necessarily be elevated at the time of a blood draw due to fasting vs nonfasting state and there may be a greater rise toward the later weeks of pregnancy. The measurement of bile acid levels in the blood is diagnostic, but it is not commonly available.

According to RCOG Guideline No. 43, April 2011 "Obstetric cholestasis is diagnosed when otherwise unexplained pruritus occurs in pregnancy and abnormal liver function tests (LFT) and/ or raised bile acids occur in the pregnant woman and both resolve after delivery. Pruritus that involves the palms and soles of the feet is particularly suggestive."

Hence, in our study, we used clinical presentation of the patients and LFT tests for the diagnosis and further management.

Current pharmacological treatment includes the use of UDCA upon diagnosis of ICP. UDCA has been shown to have a greater efficacy than other treatment modalities, including the use of $S$ adenosyl-I-methionine, dexamethasone, cholestyramine, in maternal pruritus and improving total bile acids (TBA) and serum 
transaminases. In a meta analysis, it was shown that UDCA was not only effective in reducing pruritus and improving liver tests, but also improved the maternal and perinatal outcome in patients with ICP. ${ }^{15}$

In a randomized, double-blinded placebo controlled trial by Glantz et al., it was found that UDCA was more effective than dexamethasone in relieving pruritus, and improving serum biochemical markers of ICP; however, there was no effect of UDCA or dexamethasone on fetal complication rates. Mazzella et al. reported no adverse reactions of high dose UDCA (1.5-2 g/day) and concluded that the high dose UDCA treatment appeared to improve both biochemical and clinical parameters of cholestasis and seemed to be completely safe for the fetus.

Zapata et al. concluded that UDCA was well tolerated by pregnant women and no adverse effect were detected in 26 infants followed up for a mean of 12 years after delivery, and confirmed a normal growth and development. ${ }^{22}$

In our study, UDCA was used at the dose of $600-900 \mathrm{mg} /$ day and there was no significant effect on the fetus.

\section{Conclusion}

The incidence of ICP is high in our hospital. Larger studies are needed to assess the correct incidence in the general populations. Cholestasis of pregnancy has an adverse effect on the fetal outcome; therefore, early diagnosis and treatment with UDCA are essential. This provides symptomatic relief, improvement of liver function and may contributes to the improvement of perinatal outcome. Fetal outcome are improved with a variety of strategies of active management. A common practice followed in our hospital was induction of labour at 37-38 weeks of gestation, which reduced the perinatal complications. There is a significant risk of recurrence in subsequent pregnancies; so maternal education will help to prevent the perinatal complications in future pregnancies.

\section{References}

1. Kenyon AP, Girling JC. Obstetric Cholestasis. In: Studd J. ed. Progress in obstetrics and Gynaecology. Edinburg: Churchhill Livingstone; 2005. pp. 37-56.

2. UK: Royal College of Obstetricians and Gynaecologists Obstetric cholestasis Green-Top Guideline No 43; page 3 [Updated April 2011]. rcog.org [Internet] Available from http://www.rcog.org.uk

3. Kenyon AP, Piercy CN, et al. Obstetric cholestasis, outcome with active management: a series of 70 cases. Bjog-Int J Obstet Gy 2002;109:282-288. DOI: 10.1111/j.1471-0528.2002.01368.x.

4. Saleh MM, Abdo KR. Intrahepatic Cholestasis of pregnancy: review of the literature and evaluation of current evidence. J Women's Health 2007;16:833-841. DOI: 10.1089/jwh.2007.0158.

5. Glantz A, Marschall HW, et al. Intrahepatic cholestasis of pregnancy: relationships between bile acid levels and fetal complication rates. Hepatology 2004;40:467-474. DOI: 10.1002/ hep.20336.
6. Bacq Y, Sapey T, et al. Intrahepatic cholestasis of pregnancy: a French prospective study. Hepatology 1997;26:358-364. DOI: 10.1002/ hep.510260216.

7. Brites $D$, Rodrigues CMP, et al. Unusual case of severe cholestasis of pregnancy with early onset, improved by ursodeoxycholic acid administration. Eur J Obstet Gynecol Reprod Biol 1998;76:165-168. DOI: 10.1016/S0301-2115(97)00185-1.

8. Berg B, Helm G, et al. Cholestasis of Pregnancy - Clinical and Laboratory Studies. Acta Obstet Gynecol Scand 1986;65:107-113. DOI: 10.3109/00016348609158363.

9. Reyes $\mathrm{H}$. The Spectrum of Liver and Gastrointestinal-Disease Seen in Cholestasis of Pregnancy. Gastroenterol Clin N 1992;21: 905-921.

10. Shaw D, Frohlich J, et al. A Prospective-Study of 18 Patients with Cholestasis of Pregnancy. Am J Obstet Gynecol 1982;142:621-625. DOI: 10.1016/S0002-9378(16)32430-9.

11. Arrese $M$, Reyes H. Intrahepatic cholestasis of pregnancy: a past and present riddle. Ann Hepatol 2006;5:202-205. DOI: 10.1016/S16652681(19)32012-5.

12. Brites $D$, Rodrigues $C M$, et al. Relevance of serum bile acid profile in the diagnosis of intrahepatic cholestasis of pregnancy in an high incidence area: Portugal. Eur J Obstet Gynecol Reprod Biol 1998;80:31-38. DOI: 10.1016/S0301-2115(98)00086-4.

13. Tann NN, Neuberger J, et al. Liver abnormality in pregnancy. Best Pract Res Clin Gasteroenterol 2013;27:565-575. DOI: 10.1016/ j.bpg.2013.06.015.

14. Bacq $Y$, Sentilhes L, et al. Efficacy of ursodeoxycholic acid in treating intrahepatic cholestasis of pregnancy: a meta analysis. Gastroenterology 2012;143:1492-1501. DOI: 10.1053/ j.gastro.2012.08.004.

15. Grand Maison S, Durand M, et al. The effect of ursodeoxycholic acid treatment for intrahepatic cholestasis of pregnancy on maternal and fetal outcomes: a meta-analysis including nonrandomized studies. J Obstet Gynaecol Can 2014;36:632-641. DOI: 10.1016/S17012163(15)30544-2.

16. Diaferia A, Nicastri P, et al. Ursodeoxycholic acid therapy in pregnant women with cholestasis. Int J Gynecol Obstet 1996;52:133-140. DOI: 10.1016/0020-7292(95)02528-6.

17. Schultz F, Hasan A, et al. The protective effect of ursodeoxycholic acid in an in vitro model of the human fetal heart occurs via targeting cardiac fibroblasts. Prog Biophys Mol Biol 2016;120:149-163. DOI: 10.1016/j.pbiomolbio.2016.01.003.

18. Geenes V, Lövgren-Sandblom A, et al. The reversed feto maternal bile acid gradient in intrahepatic cholestasis of pregnancy is corrected by ursodeoxycholic Acid. PLoS One 2014;9:e83828. DOI: 10.1371/journal. pone. 0083828.

19. Heinonen S, Kirkinen P. Pregnancy outcome with intrahepatic cholestasis. Obstetrics and Gynecology 1999;94:189-193. DOI: 10.1016/S0029-7844(99)00254-9.

20. The journal of obstetrics and Gynecology of India. J Obstet Gynecol India May-June 2010;60(3).

21. Germain AM, Kato S, et al. Bile acid increase response and expression of human myometrial oxytocin receptor. Am J Obstet Gynaecol 2003;189:577-582. DOI: 10.1067/S0002-9378(03)00545-3.

22. Zapata R, Lorena S, et al. UDCA in the treatment of ICP of pregnancy-a 12 year experience. Liver Int 2005;25:548-554. DOI: 10.1111/j.14783231.2004.0996.x. 